\title{
Species Diversity and Antifungal Susceptibilities of Oral Yeasts from Patients with Head and Neck Cancer
}

\author{
Jinyan $\mathrm{Wu}^{1,2, *}$ \\ Chengwen $\mathrm{Gan}^{3, *}$ \\ Jingyuan $\mathrm{Li}^{1,2}$ \\ Yiwei Liu' \\ Zhongyao Chen ${ }^{2}$ \\ Yunxia Zhang ${ }^{1,2}$ \\ Guohui $Y^{1,2}$ \\ Jinlei Sui ${ }^{1,2}$ \\ Jianping $X u\left(\mathbb{D}^{2,4}\right.$
}

'Key Laboratory of Emergency and Trauma of Ministry of Education, Hainan Medical University, Haikou, 57II99, Hainan, People's Republic of China; 2Public Laboratory of Hainan Medical University, Haikou, 57I 199, Hainan, People's Republic of China; ${ }^{3}$ Department of Oral Maxillofacial Surgery, Hainan General Hospital, Haikou, Hainan, 570203, People's Republic of China; ${ }^{4}$ Department of Biology, McMaster University, Hamilton, Ontario, L8S 4KI, Canada

*These authors contributed equally to this work
Purpose: To investigate the colonization and susceptibility to antifungal drugs of oral yeasts in head and neck cancer patients in Hainan, China.

Methods: Oral mucosa samples from 211 head and neck cancer patients were collected. Oral yeasts were isolated and identified to species by rDNA ITS sequencing. The susceptibilities of all yeasts to amphotericin B, fluconazole, fluorocytosine, itraconazole, and ketoconazole were determined.

Results: Yeasts were isolated from 124 of the 211 oral swabs. The 124 yeast isolates were classified into following 10 species, from the most frequent to the least frequent, Candida albicans (53.2\%), Candida tropicalis (22.6\%), Candida krusei (6.5\%), Kodamaea ohmeri (5.6\%), Candida parapsilosis (4.8\%), Hanseniaspora opuntiae (2.4\%), Candida metapsilosis (1.6\%), Pichia terricola (1.6\%), Pichia norvegensis (0.8\%), and Trichosporon asahii $(0.8 \%)$. The overall frequencies of resistance among the yeasts to amphotericin $\mathrm{B}$, fluconazole, flucytosine, itraconazole, and ketoconazole were $4.8 \%, 8.1 \%, 16.1 \%, 9.7 \%$, and $9.7 \%$, respectively. One $C$. albicans strain and one C. tropicalis strain were tolerant/resistant to all five drugs.

Conclusion: Given the high prevalence of oral yeast colonization in head and neck cancer patients and the observed resistance of certain yeast isolates to the five antifungal drugs, our results suggest that rapid identification and susceptibility testing should be implemented before antifungal treatment is applied among patients with head and neck cancer in Hainan. Keywords: head and neck cancer, oral yeast, Candida, antifungal resistance

\section{Introduction}

Head and neck cancer is among the most frequent cancers worldwide. ${ }^{1,2}$ In addition to its high prevalence, the survival rate for patients with head and neck cancer especially oral cancer is very low - even with the best care, the five-year survival rate rarely reached $50 \%{ }^{3}$ While advances in surgical technology have improved the patients' quality of life after surgery, according to meta-analyses of patient data over the past 50 years, the overall mortality rate has not changed. ${ }^{4}$ The commonly used treatment for head and neck cancer patients is local radiotherapy, which often lead to imbalance of the oral microbial flora, resulting in increased mucosal infections of the oral cavity as well as systemic infections by opportunistic yeast pathogens.

Most yeast pathogens are opportunistic pathogens. They are among the common components of the commensal microflora in humans. They are frequently found on
Correspondence: Jianping $\mathrm{Xu}$ Department of Biology, McMaster University, 1280 Main St. West, Hamilton, Ontario, L8S 4KI, Canada

Tel $+|-905-525-9| 40 \times 27934$

Fax + I-905-522-6066

Email jpxu@mcmaster.ca 
the skin, oral mucosa and gastrointestinal tract. In most healthy hosts, these commensal yeasts do not cause disease or pathophysiological damages to host tissues and organs. However, in immunocompromised hosts, these commensal yeasts can cause a variety of infections, from superficial, subcutaneous, to invasive infections in many parts of the body. ${ }^{5,6}$ Indeed, fungal infections caused by opportunistic yeast pathogens especially Candida species have become a major problem for healthcare systems around the world, leading to significant mortality and high economic burden for both patients and governments. $^{6-12}$ At present, at least 15 Candida species are known to cause human infectious diseases, with the following five as the main pathogenic species: Candida albicans, Candida krusei (syn. Pichia kudriavzevii and Issatchenkia orientalis), Candida parapsilosis, Candida tropicalis, and Candida glabrata (syn. Torulopsis glabrata). ${ }^{6-11}$

The major Candida pathogens (and most human fungal pathogens) differ from each other in their intrinsic susceptibilities to several common antifungal drugs. However, there have been increasing reports of acquired drug resistance among human fungal pathogens, including those in the genus Candida. Part of the increase in drug resistance has been linked to the widespread application of antifungals in both agricultural and clinical settings. The drug-resistant species and strains are complicating clinical treatments. Identifying yeast species distribution and their antifungal drug susceptibility patterns can help develop effective prevention and treatment strategies against yeast pathogens.

Within the human microbiome, the oral cavity represents a significant niche, containing hundreds of microbial species. ${ }^{13}$ The oral cavity is also a gateway for microorganisms to enter many other human body sites. Though the majority of the microbial species in the oral cavity are prokaryotic, opportunistic yeast pathogens are also commonly found on the oral mucosa. Over the last several decades, infections caused by opportunistic yeast pathogens have become the most prevalent fungal infections in humans. Moreover, intrinsically drug tolerant/resistant species have become more common, likely driven by the widespread application of antifungal drugs. ${ }^{14-19}$ However, because the oral cavity in humans mostly serves as a transient entry site for food, drinks, and medicine (only for patients taking oral drugs), the oral cavity is often not considered a niche where antifungal resistance is selected for yeast pathogens. Consequently, the origins of drug resistance among oral microbes remain largely unknown.
Since most human pathogenic fungi, including those in the genus Candida, have environmental ecological niches outside of humans, it's been hypothesized that the observed antifungal resistance in humans is at least partly due to selection pressure from the environment, including those of agricultural fungicides. Thus, understanding the drug susceptibility profiles of oral yeasts will not only help clinical decision-making but also facilitate developing sound agricultural and environmental policies on fungicide usages.

In cancer patients, postoperative infections caused by opportunistic yeast pathogens not only prolong the recovery time of patients but also bring huge financial and other costs. Consequently, understanding the diversity and distribution of oral yeasts and their drug susceptibility profiles among patients with head and neck cancer will have significant implications for both before and after surgery to prevent and treat yeast infections. However, at present, the oral yeast flora and their antifungal susceptibility for patients with head and neck cancer in Hainan are not known. In this study, we aim to investigate oral yeast colonization and their susceptibilities to antifungal drugs from patients with head and neck cancer in Hainan General Hospital, the largest hospital in Hainan, the southern-most province in China.

\section{Materials and Methods Samples}

We obtained oral swabs from 211 patients who were hospitalized at Hainan General Hospital in Haikou, the capital city of Hainan province and the largest city on Hainan Island in southern China. All patients with head and neck cancer treated at the hospital in 2017 and 2018 were recruited for this study. Our sampling and study were approved by the Ethics Committees of Hainan General Hospital and Hainan Medical University. This study was conducted in accordance with the Declaration of Helsinki. This study did not impose any medical intervention on any patients. All patients were provided information about the study and all signed a consent form about their willingness to participate. The swabs were taken in 2017 and 2018 . Among the 211 patients who agreed to participate, 72 were from the Radiotherapy Division and 139 from the Dentalcare Division, including a total of 144 male and 67 female patients (Table 1). The patients were mainly distributed in the 41 to 60 years old age group. All 211 patients were diagnosed and being treated for head or neck 
cancer at the time of sampling. The number of patients with each type of cancer or a combination of cancers is summarized in Table 1. For the 72 patients from the Radiotherapy Division, all had undergone one round of radiation treatment before oral swabs were taken for yeast isolation. At the time when the swabs were taken, no patient had any clinical signs of yeast infection in the oral cavity and none had taken any antifungal drug within three months or used any mouthwash within at least two hours up to the sampling. Oral swab collections and the isolations of yeasts from these swabs all followed previously described protocols. ${ }^{20,21}$ Briefly, sterile cotton swabs were used to sample the whole upper and lower outer gingiva of each person. After sampling, the tip of each swab was immediately cut off by a sterilized pair of scissors and submerged in a $2 \mathrm{~mL}$ sterile cryogenic tube containing $1 \mathrm{~mL}$ sterilized yeast extract-peptone-dextrose (YEPD: $1 \%$ yeast extract, $2 \%$ peptone, $2 \%$ dextrose) broth with $50 \mathrm{mg} / \mathrm{L}$ of the antibiotic chloramphenicol. The tubes were taken to the lab and incubated at $30^{\circ} \mathrm{C}$ for three days. The broths were then streaked onto YEPD agar (YEPD + $2 \%$ agar) to select for yeast colonies. Distinct yeast colonies were then purified by further streaking onto new YEPD agar. All purified yeasts isolated from these swabs

Table I Carriage and Distribution of Yeast Species from the Oral Swabs Among Head and Neck Cancer Patients in Hainan, China

\begin{tabular}{|c|c|c|c|c|c|c|c|c|c|c|c|c|c|}
\hline Host Trait & Host Groups & $\begin{array}{c}\text { No. of } \\
\text { Patients }\end{array}$ & $\begin{array}{c}\text { No. of Patients with } \\
\text { Yeasts (\% Positive) }\end{array}$ & $\mathrm{Ca}$ & $C t$ & $C_{k}$ & Ko & Cp & Ho & $\mathbf{P t}$ & $\mathrm{Cm}$ & $\mathbf{T a}$ & Pn \\
\hline \multirow[t]{2}{*}{ Age group } & $\leq 59$ years old & 150 & $82(54.7)$ & 43 & 16 & 6 & 7 & 3 & 2 & 2 & I & I & I \\
\hline & $\geq 60$ years old & 61 & $42(68.9)$ & 23 & 12 & 2 & - & 3 & I & - & I & - & - \\
\hline \multirow[t]{2}{*}{ Gender } & Male & 144 & $84(58.3)$ & 51 & 15 & 6 & 5 & 3 & 2 & 2 & - & - & - \\
\hline & Female & 67 & $40(59.7)$ & 15 & 13 & 2 & 2 & 3 & I & 0 & 2 & 1 & I \\
\hline \multirow[t]{2}{*}{$\begin{array}{l}\text { Treatment } \\
\text { condition }\end{array}$} & $\begin{array}{l}\text { Without local } \\
\text { radiotherapy }\end{array}$ & 139 & $8 I(58.3)$ & 42 & 20 & 3 & 5 & 3 & 2 & 2 & 2 & 1 & I \\
\hline & With local radiotherapy & 72 & $43(59.7)$ & 24 & 8 & 5 & 2 & 3 & I & - & - & - & - \\
\hline \multirow{22}{*}{$\begin{array}{l}\text { Types of } \\
\text { cancer }\end{array}$} & Tongue cancer & 32 & $24(75)$ & 15 & 6 & - & - & I & I & 1 & - & - & - \\
\hline & $\begin{array}{l}\text { Nasopharyngeal } \\
\text { carcinoma }\end{array}$ & 63 & $37(58.7)$ & 19 & 9 & 2 & 2 & 3 & - & 1 & - & 1 & - \\
\hline & Craniopharyngioma & 2 & $I(50.0)$ & - & 1 & - & - & - & - & - & - & - & - \\
\hline & Lip cancer & 5 & $I(20.0)$ & - & I & - & - & - & - & - & - & - & - \\
\hline & $\begin{array}{l}\text { Tongue squamous cell } \\
\text { carcinoma }\end{array}$ & 6 & $0(0.0)$ & - & - & - & - & - & - & - & - & - & - \\
\hline & Brain cancer & 7 & $5(71.4)$ & 3 & I & I & - & - & - & - & - & - & - \\
\hline & Basal cell carcinoma & 2 & $I(50.0)$ & I & - & - & - & - & - & - & - & - & - \\
\hline & Laryngeal cancer & 7 & $6(85.7)$ & 2 & 2 & - & - & I & I & - & - & - & - \\
\hline & Cheek cancer & 10 & $9(90.0)$ & 5 & 2 & - & 1 & - & I & - & - & - & - \\
\hline & Lip and Cheek cancer & 2 & $2(100.0)$ & I & I & - & - & - & - & - & - & - & - \\
\hline & Esophageal cancer & 3 & $3(100.0)$ & 3 & - & - & - & - & - & - & - & - & - \\
\hline & Gum cancer & 7 & $4(57.1)$ & 3 & I & - & - & - & - & - & - & - & - \\
\hline & Parotid gland cancer & 6 & $2(33.3)$ & - & I & - & I & - & - & - & - & - & - \\
\hline & $\begin{array}{l}\text { Pyriform fossa } \\
\text { squamous cell } \\
\text { carcinoma }\end{array}$ & I & $\mathrm{I}(100.0)$ & - & - & I & - & - & - & - & - & - & - \\
\hline & Pharyngeal Cancer & 7 & $2(28.6)$ & 2 & - & - & - & - & - & - & - & - & - \\
\hline & Multiple oral cancer & I & $I(100.0)$ & - & - & 1 & - & - & - & - & - & - & - \\
\hline & Right palate cancer & 5 & $4(80.0)$ & 1 & - & - & 3 & - & - & - & - & - & - \\
\hline & Neck cancer & 8 & $4(50.0)$ & 2 & - & 1 & - & - & - & - & - & - & 1 \\
\hline & Left cheekbone mass & I & $I(100.0)$ & - & - & I & - & - & - & - & - & - & - \\
\hline & $\begin{array}{l}\text { Submandibular } \\
\text { adenocarcinoma }\end{array}$ & 6 & $0(0.0)$ & - & - & - & - & - & - & - & - & - & - \\
\hline & Mandibular bone cancer & 15 & $9(60.0)$ & 5 & - & I & - & I & - & - & 2 & - & - \\
\hline & Maxillary bone cancer & 14 & $7(50.0)$ & 5 & 2 & - & - & - & - & - & - & - & - \\
\hline
\end{tabular}

Abbreviations: Ca, Candida albicans; Ct, Candida tropicalis; Ck, Candida krusei; Ko, Kodamaea ohmeri; Cp, Candida parapsilosis; Ho, Hanseniaspora opuntiae; Pt, Pichia terricola; $C m$, Candida metapsilosis; Ta, Trichosporon asahii; Pn, Pichia norvegensis. 
have been stored in Sabouraud dextrose broth containing $30 \%$ glycerol in a $-80{ }^{\circ} \mathrm{C}$ freezer.

\section{Identification of Yeast Species}

To identify the yeasts, we first retrieved the stored isolates from the $-80^{\circ} \mathrm{C}$ freezer and plated them on YEPD agar medium. The plates were incubated for $48-72 \mathrm{~h}$ at $30^{\circ} \mathrm{C}$. We then harvested the actively growing yeast cells and extracted their genomic DNA followed the protocol described previously by $\mathrm{Xu}$ et al. ${ }^{22}$ To identify the species affiliation of these yeast isolates, we used the universal fungal primers ITS1 and ITS4 to amplify the intertranscribed spacer (ITS) regions of the nuclear ribosomal RNA gene cluster through polymerase-chain reaction (PCR), following protocols described previously. ${ }^{20}$ The amplified ITS PCR products were sequenced at BGIShenzhen, China. The ITS regions are the universal fungal DNA barcode. ${ }^{23}$ As a result, ITS sequences of many known fungal species are available in GenBank as well as in other fungal taxonomy-related databases. Here, our obtained yeast ITS sequences were compared to those in the GenBank through the BLAST search option. The best match based on the full-length ITS sequence to the type strain of each known yeast species in the GenBank database was used for yeast species identification. Specifically, we used $\geq 97 \%$ nucleotide sequence identity over the full region of ITS (covering both regions 1 and 2 of the ITS) and a BLAST E-score of $<10^{-10}$ as cutoffs. ${ }^{20,21}$ In addition, we confirmed the species identity by comparing with those in the medical fungi ITS barcode database (https:// its.mycologylab.org/) using BLAST searches.

\section{Antifungal Susceptibility Testing}

For all yeast isolates from these patients, we determined their susceptibility to five common antifungal agents: amphotericin B, fluorocytosine, itraconazole, fluconazole, and ketoconazole. These five antifungal drugs represent three distinct targets in fungal pathogens. These five agents are among the most commonly used antifungal drugs in China for treating yeast infections. ${ }^{24,25}$ We used the agar disk diffusion method to determine their susceptibility, following the CLSI M44-A2 guidelines. ${ }^{26}$ Disks containing these five drugs were all obtained from $\mathrm{ROSCO} .{ }^{27} \mathrm{We}$ used the reference strains as recommended in CLSI M44$\mathrm{A} 2^{26}$ and followed the interpretive criteria of $\mathrm{ROSCO}^{27}$ for susceptible (S), intermediate (I), and resistance (R) for each of the five drugs. The detailed procedures for determining susceptibility have been described in previous studies. ${ }^{20,28}$

\section{Analyses of Data}

We compared the yeast carriage rates between different demographic groups of head and neck cancer patients, using the relevant functions in the Microsoft Excel program. ${ }^{29}$ Specifically, we investigated whether the following four host features (Table 1) contributed to yeast carriage rate differences: age ( $60 \mathrm{yr}$ and older vs $59 \mathrm{yr}$ and younger), sex (female vs male), local radiotherapy (yes vs no), and 22 types of head and neck cancer or combinations of head and neck cancer types (tongue cancer, nasopharyngeal carcinoma, etc. Table 1). Aside from analyzing the overall yeast carriage rate differences, we also compared yeast species distributions among oral swab samples from host groups using the Chi-square test. ${ }^{29}$ In the case of small number of patients in a specific cancer group, Fisher's Exact test was used. However, due to the small numbers of isolates for most yeast species in our population samples (see below), we only compared the potential differences in yeast species distributions among different demographic groups for the two most common species, C. albicans and C. tropicalis (see below).

\section{Results}

Among the 211 swabs from the oral cavities of 211 head and neck cancer patients, we successfully isolated yeasts from 124 of them, yielding an overall 58.8\% isolation rate for yeasts. Table 1 summarizes the yeast carriage rates in the oral cavity among patient groups from Hainan General Hospital, of different sexes, ages, treatments (ie, with or without radiotherapy) and different types of cancer. Our analyses revealed no statistically significant difference in yeast isolation rates between the two treatment groups with or without radiotherapy $(\mathrm{P}=0.839)$ and between the two sexes $(\mathrm{P}=0.849)$. However, older patients (60 $\mathrm{yr}+)$ had a significantly higher oral yeast carriage rate $(68.9 \%)$ than younger patients (59 $\mathrm{yr}$ and younger) $(54.7 \%)(\mathrm{P}=0.05)$. Significant differences were also found among the 14 patient groups with different types of cancer ( $\mathrm{P}<0.001$, only patient groups with five or more patients were included in the analyses). Among the cancer groups with five or more patients, those with cheek cancer had the highest yeast carriage rate $(9 / 10 ; 90 \%)$. In contrast, no yeast was isolated in patients with tongue squamous cell carcinoma (0/6) and those with submandibular adenocarcinoma $(0 / 6)$. Oral swabs from the following five 
patient groups all contained yeasts: lip and cheek cancer, esophageal cancer, pyriform fossa squamous cell carcinoma, left cheekbone mass, and multiple oral cancers. However, each of these patient groups had only one to three patients each. The yeast carriage rates among the 14 remaining cancer patient groups were between $20 \%$ and $87.5 \%$ (Table 1).

We found no statistically significant difference in species composition between oral yeast samples from the two age groups $(\mathrm{P}=0.462)$ or among patient groups with different types of cancer(s) $(\mathrm{P}=0.685)$ (Table 1). However, yeast populations from the two sexes differed significantly in their yeasts species distributions ( $\mathrm{P}=$ 0.021). Specifically, the samples from males analyzed here had a higher percentage of C. albicans (60.7\%) than those from females $(37.5 \%)$. In contrast, samples from females had a higher percentage of C. tropicalis (32.5\%) than those from male (17.6\%).

\section{Distribution of Yeast Species}

Based on ITS sequences, the 124 yeast isolates were classified into 10 species. Based on their relative frequencies, the overall prevalence of each of the 10 species, from the most common to the least common in our sample, was $C$. albicans (66 isolates, 53.2\%), C. tropicalis (28, 22.6\%), C. krusei (8, $6.5 \%)$, Kodamaea ohmeri (7, 5.6\%), C. parapsilosis (6, 4.8\%), Hanseniaspora opuntiae (3, 2.4\%), Pichia terricola (2, 1.6\%), C. metapsilosis (2, 1.6\%), Trichosporon asahii (1, $0.8 \%$ ), and Pichia norvegensis (1,0.8\%). In 11 of the 22 cancer groups, the most prevalent yeast species was C. albicans. Interestingly, $K$. ohmeri was the most frequent species isolated from patients with right palate cancer (3/4). For the remaining patient groups, the sample sizes were very small and other species may be dominant.

\section{Profile of Antifungal Susceptibilities}

Results of our antifungal susceptibility testing of all the oral yeasts are summarized in Table 2. The frequencies of isolates with intermediate (I) susceptible and/or resistant (R) phenotypes varied widely among the yeast species as well as among the five antifungal agents (Table 2). Overall, six of the 10 yeast species had at least one isolate with the I and/or the R phenotypes to at least one of the five antifungal drugs. The remaining four yeast species (C. parapsilosis, H. opuntiae, C. metapsilosis and $P$. norvegensis) representing twelve isolates total did not have either the I or the R phenotype against any of the five drugs. However, resistance to four of the five antifungal agents (except amphotericin B) was observed for several strains of C. krusei. Significantly, two of the 124 yeast isolates were not sensitive to any of the five antifungal agents tested in this study. One was a $C$. albicans isolate obtained from a $57 \mathrm{yr}$ old male with nasopharyngeal carcinoma treated by the Radiotherapy Division. The other isolate belonged to C. tropicalis and was obtained from a $50 \mathrm{yr}$ old male with craniopharyngioma also treated by the Radiotherapy Division (Table 2).

Overall, 4.8\% (6/124) of all yeast strains were resistant to amphotericin B, 8.1\% (10/124) resistant to fluconazole, 9.7\% (12/124) resistant to itraconazole, 9.7\% (12/124) resistant to ketoconazole, and $16.1 \%(20 / 124)$ resistant to fluorocytosine. However, none of the host demographic factors or underlying disease condition was associated with the frequencies of antifungal drug resistance (detailed analyses not shown).

\section{Discussion}

The oral cavity is an important ecological niche and a major route of entry for microbial pathogens into the human digestive tract and other organ systems. Infections of the oral cavity are closely related to the overall health status of the host and to mortality. ${ }^{30,31}$ In addition, Candida infection has been associated with the development of cancer. ${ }^{32}$ For example, a Danish report estimated that patients infected with Candida had two-fold increase in cancer risks in the esophagus, oropharynx, and tongue. ${ }^{33}$ For head and neck cancer patients, especially those undergoing radiotherapy, their immune system is often compromised. Consequently, this group of patients are especially susceptible to oral mucosal and systemic yeast infections. Therefore, understanding their yeast flora, including susceptibilities to antifungal drugs, is extremely important to help prevent and treat such infections.

Our study collected a total of 211 oral mucosal specimens from patients with head and neck cancer. Among these, we were able to isolate yeasts from 124 (58.8\%) patients. This yeast isolation rate is similar to that of the Emergency Department patients and the general patient population from several hospitals $(66.7 \%)$ in Hainan, as reported in a previous study. ${ }^{20}$ However, the yeast isolation rates from the different groups of patients in Hainan were all much higher than that of healthy people in Hainan $(23.1 \%) .{ }^{21}$ In addition, we found that older patients $(60 \mathrm{yr}$ + ) with head and neck cancer had a significantly higher rate of oral yeast colonization (68.9\%) than those younger 
Table 2 Summary Susceptibilities of Oral Yeasts from Head and Neck Cancer Patients in Hainan to Five Antifungal Agents

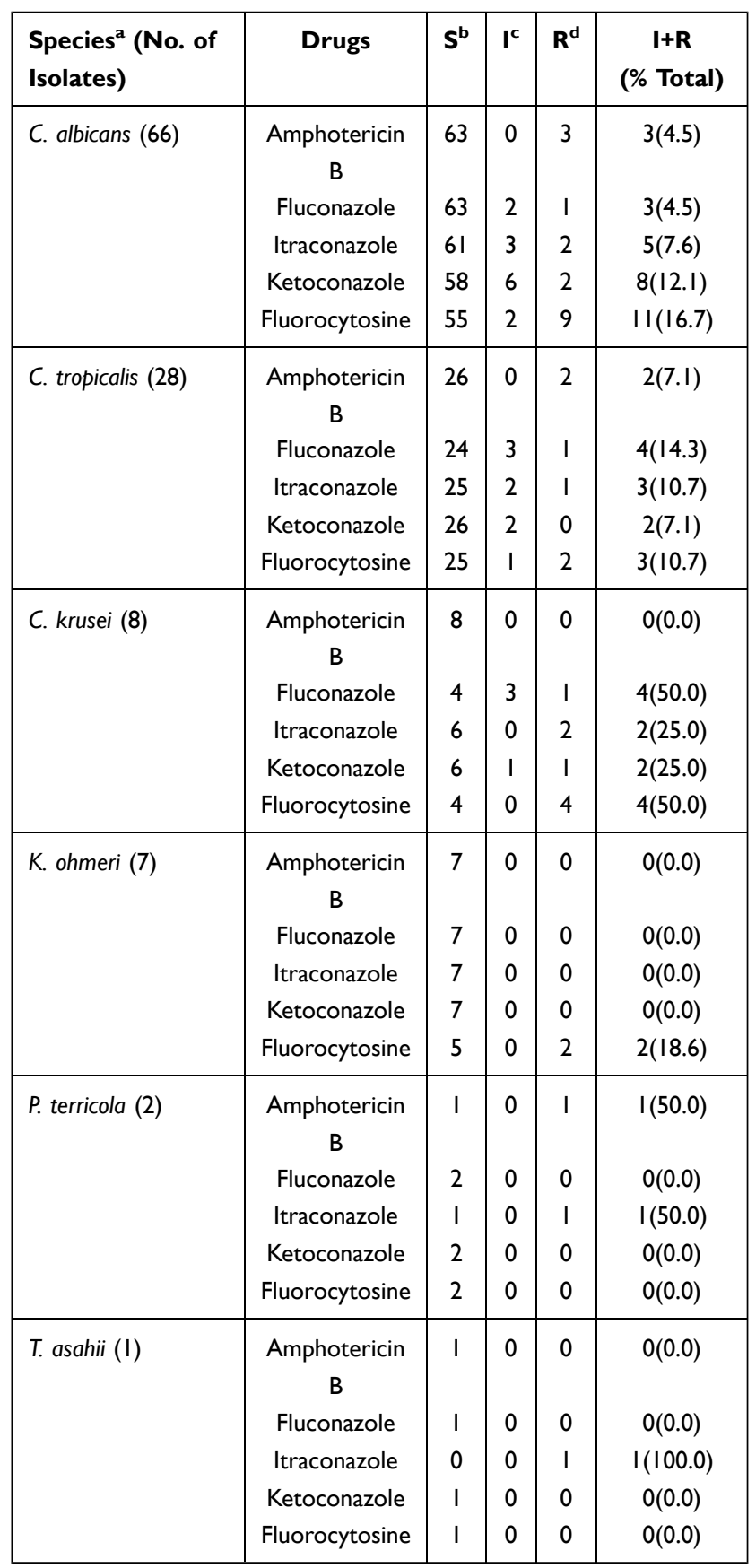

Notes: ${ }^{a}$ For the remaining four yeast species, C. parapsilosis (6), H. opuntiae (3), C. metapsilosis (2) and $P$. norvegensis ( 1 ), all isolates were sensitive to all five tested antifungal agents. ${ }^{b}$, susceptible to drug, ${ }^{\mathrm{C}}$, intermediate susceptible to drug, ${ }^{\mathrm{d}} \mathrm{R}$, resistant to drug.

patients $(54.7 \%)(\mathrm{P}=0.05)$. This observed age-group difference was similar to earlier reports for both healthy hosts and other types of patients in Hainan. ${ }^{20,21}$ While we did not record denture wear among patients, it's possible that older patients may have a higher frequency of denture wear usage in Hainan that could have contributed to their higher frequency of Candida colonization. Together, these studies suggest that old age and compromised health are both associated with high oral yeast carriage rate in the Hainan population.

Previous studies have shown that Candida infections in cancer patients in Europe were mainly caused by three species, C. albicans, C. tropicalis and C. glabrata. $^{34}$ Here, among the 124 yeast strains, C. albicans (53.2\%) and $C$. tropicalis $(22.6 \%)$ were the two most common yeast species, similar to those found in Europe. Indeed, in most epidemiological surveys reported so far around the world, C. albicans is the most prevalent yeast species associated with humans, either as a commensal or as an opportunistic pathogen. Similarly, C. tropicalis accounts for a significant proportion of clinical candidiasis worldwide. This is especially true for several geographic areas such as Brazil and East Asia. In these regions, C. tropicalis is either the first or the second most frequently isolated yeasts in humans. ${ }^{15,35,36}$ The high-frequency isolation of C. tropicalis in our current study is consistent with the global pattern for tropical regions.

However, though C. glabrata is a commonly found yeast in humans, none of the 124 yeasts in our sample belonged to this species. Instead, C. krusei (=Pichia kudriavzevii) was the third most common species (6.5\%) in this sample. C. krusei is commonly found in the production of fermented foods, including chocolate, drinks such as liquor, and preserved vegetables. ${ }^{37}$ It's tempting to speculate that the C. krusei isolates from among the head and neck cancer patients in our study likely originated in the foods, drinks, or the immediate environments of these patients. Indeed, phylogenomic analysis of clinical and environmental isolates revealed close relationships between those ecological populations with no clear separation of clinical and environmental isolates of C. krusei into distinct subpopulations. ${ }^{37}$ Together, these results revealed the importance of analyzing environmental populations of yeasts, including those in our foods, for understanding the epidemiology of yeast infections in humans. Our results suggest that care should be taken to ensure protection for people with compromised immunity who works in certain food production fields where opportunistic pathogenic yeasts such as $C$. krusei may be prevalent. Similar cautions may be extended to consumers with weakened immune functions about foods derived from fermentations of such opportunistic yeast pathogens.

The fourth most common species $(5.6 \%)$ in this sample was $K$. ohmeri. K. ohmeri (previously known as Pichia 
ohmeri or Yamadazyma ohmeri) belong to the same family of yeasts as Candida. ${ }^{38,39}$ In the last two decades, it has been recognized as an emerging yeast pathogen that can cause a diversity of infections in both immunocompromised and some apparently immunocompetent humans, including fungemia, funguria, endocarditis, peritonitis and wound infections. ${ }^{39-42}$

C. parapsilosis was the fifth most common species $(4.8 \%)$ in this study. Similar to $C$. albicans and C. tropicalis, C. parapsilosis can cause a diversity of yeast infections in both immunocompromised and immunocompetent hosts. Of special note is that $C$. parapsilosis can form abundant biofilms on plastic and other artificial surfaces and is a common asymptomatic colonizer of the human skin and mucosal surfaces. In addition, it can grow rapidly in parenteral nutrition administered to ICU patients, thereby placing undernourished children and lowbirth-weight neonates ${ }^{43-45}$ at increased risk.

The other five species were $H$. opuntiae $(3,2.4 \%)$, P. terricola (2, 1.6\%), C. metapsilosis (2, 1.6\%), T. asahii (1, 0.8\%), and P. norvegensis (1, 0.8\%). $H$. opuntiae and P. terricola can be isolated from soil, fruit juice, sea water and other habitats and shows many similarities to P. kudriavzevii, with both capable of causing systemic infection. ${ }^{41,46}$ C. metapsilosis is closely related to C. parapsilosis and can cause similar infections as C. parapsilosis. T. asahii and P. norvegensis have recently been recognized as emerging fungal pathogens able to cause invasive infections in neutropenic cancer patients as well as in critically ill patients submitted to invasive medical procedures and broad-spectrum antibiotic therapy. $^{47,48 .}$

Our antifungal susceptibility testing found a number of yeast isolates being resistant to each of the five antifungal drugs, including two isolates being tolerance/resistant to all five drugs. Jahanshiri et $\mathrm{al}^{49}$ found that a large number of yeasts from patients who had undergone head and neck radiotherapy and chemotherapy were highly resistant to fluconazole and itraconazole, including cross-resistance to both drugs. Cross-resistance to multiple triazoles (ie, fluconazole, itraconazole and ketoconazole) was also found in this study. However, no statistically significant difference was found in triazole resistance rates between hosts with vs those without localized radiotherapy. Surprisingly, despite none of our patients had taken any antifungal drug prior to the sampling, the rates of amphotericin $\mathrm{B}$ resistance to $(4.8 \%)$ and flucytosine resistance $(16.1 \%)$ were higher than those of bloodstream yeast isolates reported previously from Asia, Europe, and Americas. $^{5-12,16,17}$ Those bloodstream isolates reported in the literature were mostly isolated from patients who had taken antifungal drugs. ${ }^{5-12,16,17}$ The only report that showed a higher frequency of drug resistance than ours was the recent study by Jahanshiri et al. ${ }^{49}$ Their study investigated 160 patients with neck and head cancer from Iran. ${ }^{49}$ In their survey, the three dominant species of yeasts were C. albicans $(53.3 \%), C$. tropicalis $(21.66 \%)$ and C. glabrata (15\%). The percentages of their C. albicans strains being resistant to amphotericin $\mathrm{B}$, fluconazole, ketoconazole, and itraconazole were $87.50 \%, 62.5 \%$, $93.75 \%$, and $81.25 \%$, respectively. Furthermore, their nonalbicans Candida strains also showed high frequencies of resistance to amphotericin B (69.28\%), fluconazole (42.85), ketoconazole (89.28\%), and itraconazole $(85.71 \%) .{ }^{49}$ These drug-resistant rates in Iran were much higher than the rates so far reported in any other survey, including those in our samples. One potential reason for the difference between our study and that by Jahanshiri et $\mathrm{al}^{49}$ is that our patient population in the Radiotherapy Division only underwent one round of radiotherapy before oral swabs were taken. Additional and frequent radiotherapy may cause more damages to the oral microbial defense and more mutations in the oral yeasts that could potentially contribute to increased yeast carriage rate and increased resistance to antifungal drugs, when compared with patients without any radiotherapy.

Within each of the three non-Candida species in our sample (K. ohmeri, P. terricola, T. asahii), at least one isolate showed either the I and/or the R phenotype to at least one of the five antifungal agents. This result is similar to what was reported previously for oral yeasts from hospitalized patients with other underlying conditions. ${ }^{20}$ However, this result is different from what was found in Taiwan where all their non-Candida oral yeasts were all susceptible to their tested antifungal drugs. ${ }^{50}$ We would like to stress that the sample sizes for the majority of the non-Candida oral yeast species analyzed so far have been very small. Consequently, it is difficult to determine whether the observed differences between our samples and those published previously are statistically significant. A number of hypotheses have been suggested to explain potential origin(s) of drug-resistant yeasts in patients who had never taken any antifungal drugs, ${ }^{20}$ including heavy use of agricultural fungicides and the presence of other stress factors. These hypotheses can be applied equally to 
explain the observations here, and they are not described further.

Radiotherapy is among the most frequently used methods to treat patients suffering head and neck cancer. The rate of oral fungal infections may increase in these patients because radiotherapy often leads to reduced host immunity. Oral yeast infection has been frequently associated with morbidity in these patients. These infections are commonly manifested with symptoms such as oral thrush, angular cheilitis and erythematous but can expand to other body sites such as esophagus and the blood circulatory system, leading to life-threatening systemic infections. ${ }^{51,52}$ Therefore, understanding the distribution of oral yeasts in patients with head and neck cancer and the susceptibilities of these yeasts to antifungal drugs could be of great practical significance for developing targeted preoperative and postoperative prevention and treatment strategies against yeast infections in these patients.

In conclusion, our study provides the first description of oral yeast species distribution among head and neck cancer patients in Hainan Province in southern China. In addition, we determined the patterns of antifungal susceptibilities for all 124 yeast isolates. We analyzed the potential contributions of patient age, sex, underlying head and neck cancer type, and/or radiation therapy to yeast species distribution and antifungal susceptibilities. The identification of multi-drug resistant yeasts in patients who had never taken any antifungal drugs calls for greater research efforts to monitor and track drug-resistant pathogens in our clinics and environments.

\section{Abbreviations}

rDNA, ribosome deoxyribonucleic acid; ITS, intertranscribed spacer; YEPD, yeast extract-peptone-dextrose; RNA, ribonucleic acid; PCR, polymerase-chain reaction; CLSI, Clinical Laboratory Standard Institute; R, resistant; I, intermediate; S, susceptible; C. albicans, Candida albicans; C. krusei, Candida krusei; C. metapsilosis, Candida metapsilosis; C. parapsilosis, Candida parapsilosis; C. tropicalis, Candida tropicalis; H. opuntiae, Hanseniaspora opuntiae; K. ohmeri, Kodamaea ohmeri; P. norvegensis, Pichia norvegensis; P. terricola, Pichia terricola; T. asahii, Trichosporon asahii.

\section{Data Sharing Statement}

The detailed host demographic information for each individual yeast isolate is available upon request.

\section{Acknowledgments}

We thank XIAO Jun, MO Meizi, and WANG Lulu for helping with sample collections.

\section{Funding}

This project was supported by Natural Science Foundation of Hainan Province (Grant No. 2019RC227), National Natural Science Foundation of China (Grant No. 31860035), and College Student Innovation Project (Grant No. 201911810006).

\section{Disclosure}

We declare that there are no competing financial or other interests associated with this study.

\section{References}

1. Bray F, Ferlay J, Soerjomataram I, Siegel RL, Torre LA, Jemal A. Global cancer statistics 2018: GLOBOCAN estimates of incidence and mortality worldwide for 36 cancers in 185 countries. CA Cancer J Clin. 2018;68(6):394-424. doi:10.3322/caac.21492

2. Siegel RL, Miller KD, Jemal A. Cancer statistics, 2020. CA Cancer J Clin. 2020;70(1):7-30. doi:10.3322/caac.21590

3. Johnson NW, Warnakulasuriya S, Gupta PC, et al. Global oral health inequalities in incidence and outcomes for oral cancer causes and solutions. Adv Dent Res. 2011;23(2):237-246. doi:10.1177/ 0022034511402082

4. Casiglia J, Woo SB. A comprehensive review of oral cancer. Gen Dent. 2001;49(1):72-82.

5. Mushi MF, Bader O, Taverne-Ghadwal L, Bii C, Gro U, Mshana SE. Oral candidiasis among African human immunodeficiency virus-infected individuals: 10 years of systematic review and meta-analysis from Sub-Saharan Africa. J Oral Microbiol. 2017;9 (1):1317579. doi:10.1080/20002297.2017.1317579

6. Kullberg BJ, Arendrup MC, Campion EW. Invasive candidiasis. $N$ Engl $J$ Med. 2015;373(15):1445-1456. doi:10.1056/ NEJMra1315399

7. Magill SS, Edwards JR, Bamberg W, et al. Multistate point-prevalence survey of health care-associated infections. $N$ Engl J Med. 2014;370(13):1198-1208. doi:10.1056/NEJMoa1306801

8. McCarty TP, Pappas PG. Invasive candidiasis. Infect Dis Clin North Am. 2016;30(1):103-124. doi:10.1016/j.idc.2015.10.013

9. Pappas PG, Rex JH, Lee J, et al. A prospective observational study of candidemia: epidemiology, therapy, and influences on mortality in hospitalized adult and pediatric patients. Clin Infect Dis. 2003;37 (5):634-643. doi:10.1086/376906

10. Nami S, Mohammadi R, Vakili M, Khezripour K, Mirzael H, Morovati H. Fungal vaccines, mechanism of actions and immunology: a comprehensive review. Biomed Pharm. 2018;109:333-344. doi:10.1016/j.biopha.2018.10.075

11. Wisplinghoff H, Bischoff T, Tallent SM, Seifert H, Wenzel RP, Edmond MB. Nosocomial bloodstream infections in US hospitals: analysis of 24,179 cases from a prospective nationwide surveillance study. Clin Infect Dis. 2004;39(3):309-317. doi:10.1086/421946

12. Cleveland AA, Harrison LH, Farley MM, et al. Declining incidence of candidemia and the shifting epidemiology of Candida resistance in two US metropolitan areas, 2008-2013: results from populationbased surveillance. PLoS One. 2015;10(3):e0120452. doi:10.1371/ journal.pone. 0120452 
13. Nelson KE, Madupu R, Szpakowski S, Goll JB, Krampis K, Methe BA. Next-generation sequencing, metagenomes, and the human microbiome. In: Xu J, editor. Next-Generation Sequencing: Current Technologies and Applications. Norfolk: Caister Academic Press; 2014:141-155.

14. Brown GD, Denning DW, Gow NA, Levitz SM, Netea MG, White TC. Hidden killers: human fungal infections. Sci Transl Med. 2012;4(165):165rv13. doi:10.1126/scitranslmed.3004404

15. Pristov KE, Ghannoum MA. Resistance of Candida to azoles and echinocandins worldwide. Clin Microbiol Infect. 2019;25 (7):792-798. doi:10.1016/j.cmi.2019.03.028

16. Pfaller MA, Diekema DJ. Epidemiology of invasive candidiasis: a persistent public health problem. Clin Microbiol Rev. 2007;20 (1):133-163. doi:10.1128/CMR.00029-06

17. Pfaller MA, Moet GJ, Meser SA, Jones RN, Castanheira M. Candida bloodstream infections: comparison of species distributions and antifungal resistance patterns in community-onset and nosocomial isolates in the SENTRY antimicrobial surveillance program, 2008-2009. Antimicrob Agents Chemother. 2011;55(2):561-566. doi:10.1128/ AAC.01079-10

18. Wang H, Xu YC, Hsueh PR. Epidemiology of candidemia and antifungal susceptibility in invasive Candida species in the Asia-Pacific region. Future Microbiol. 2016;11(11):1461-1477. doi:10.2217/fmb2016-0099

19. Rodloff AC, Koch D, Schaumann R. Epidemiology and antifungal resistance in invasive candidiasis. Eur J Med Res. 2011;16 (4):187-195. doi:10.1186/2047-783X-16-4-187

20. Wu J, Guo H, Yi G, et al. Prevalent drug resistance among oral yeasts from asymptomatic patients in Hainan, China. Mycopathologia. 2014;177(5-6):299-307. doi:10.1007/s11046-014-9747-3

21. Wang $\mathrm{H}, \mathrm{Xu}$ J, Guo H, et al. Patterns of human oral yeast species distribution on Hainan Island in China. Mycopathologia. 2013;176 (5-6):359-368. doi:10.1007/s11046-013-9703-7

22. Xu J, Mitchell TG, Vilgalys R. PCR-restriction fragment length polymorphism (RFLP) analyses reveal both extensive clonality and local genetic differences in Candida albicans. Mol Ecol. 1999;8 (1):59-73. doi:10.1046/j.1365-294X.1999.00523.x

23. Schoch CL, Seifert KA, Huhndorf S; The Fungal Barcode Consortium. Nuclear ribosomal internal transcribed spacer (ITS) region as a universal DNA barcode marker for Fungi. Proc Natl Acad Sci USA. 2012;109(16):6241-6246. doi:10.1073/pnas.1117018109

24. Luo C, Feng L, Dai HZ. Application of antifungal agents in 6 cities along the Yangzi River from 2005 to 2007. Chin J New Drugs Clin Remedies. 2009;10:785-790.

25. Li J. Analysis for usage of antifungal drugs from outpatient pharmacy. Strait Pharm J. 2012;24:258-259.

26. CLSI. Method for Antifungal Disk Diffusion Susceptibility Testing of Yeasts. Approved Guideline. 2nd ed. CLSI document M44-A2. Wayne, PA, USA; Clinical and Laboratory Standards Institute; 2009.

27. Anonymous. NEO-SENSITABS User's Guide in Antifungal Susceptibility Testing of Yeast. Taastrup, Denmark: A/S Rosco Diagnostica; 2011.

28. Wu JY, Guo H, Wang HM, et al. Multilocus sequence analyses reveal extensive diversity and multiple origins of fluconazole resistance in Candida tropicalis from tropical China. Sci Rep. 2017;7(1):42537. doi:10.1038/srep42537

29. Sokal RR, Rohlf FJ. Biometry: The Principles and Practices of Statistics in Biological Research. 2nd ed. New York: Freeman and Company; 1981.

30. Hämäläinen P, Meurman JH, Kauppinen M, Marja K. Oral infections as predictors of mortality. Gerodontology. 2010;22:151-157. doi:10.1111/j.1741-2358.2005.00064.X

31. Guo F, Yang Y, Kang Y, et al. Invasive candidiasis in intensive care units in China: a Multicentre Prospective Observational Study. J Antimicrob Chemother. 2013;68(7):1660-1668. doi:10.1093/jac/ $\mathrm{dkt} 083$
32. Meurman JH, Bascones-Martinez A. Are oral and dental diseases linked to cancer? Oral Dis. 2011;17(8):779-784. doi:10.1111/ j.1601-0825.2011.01837.x

33. Nørgaard M, Thomsen RW, Farkas DK, Mogensen MF, Sørensen HT. Candida infection and cancer risk: a Danish Nationwide Cohort Study. Eur J Intern Med. 2013;24(5):451-455. doi:10.1016/j. ejim.2013.02.017

34. Viscoli C, Girmenia C, Marinus A, et al. Candidemia in cancer patients: a prospective, multicenter surveillance study by the Invasive Fungal Infection Group (IFIG) of the European Organization for Research and Treatment of Cancer (EORTC). Clin Infect Dis. 1999;28(5):1071-1079. doi:10.1086/514731

35. Arrua JM, Rodrigues LA, Pereira FO, Lima EO. Prevalence of Candida tropicalis and Candida krusei in onychomycosis in João Pessoa,Paraiba, Brazil from 1999 to 2010. An Acad Bras Cienc. 2015;87(3):1819-1822. doi:10.1590/0001-3765201520130418

36. Kumar A. A fungus among us: the emerging opportunistic pathogen Candida tropicalis and PKA signaling. Virulence. 2018;9 (1):659-661. doi:10.1080/21505594.2018.1438026

37. Douglass AP, Offei B, Braun-Galleani S, et al. Population genomics shows no distinction between pathogenic Candida krusei and environmental Pichia kudriavzevii: one species, four names. PLoS Pathog. 2018;14(7):e1007138. doi:10.1371/journal.ppat.1007138

38. Yamada Y, Suzuki T, Matsuda M, Mikata K. The phylogeny of Yamadazyma ohmeri (ETCHELLS et BELL) BILLON-GRAND based on the partial sequences of $18 \mathrm{~S}$ and $26 \mathrm{~S}$ ribosomal RNAs: the proposal of Kodamaea gen. nov. (Saccharomycetaceae). Biosci Biotechnol Biochem. 1995;59(6):1172-1174. doi:10.1271/bbb.59.1172

39. Chakrabarti A, Rudramurthy SM, Kale P, et al. Epidemiological study of a large cluster of fungaemia cases due to Kodamaea ohmeri in an Indian tertiary care centre. Clin Microbiol Infect. 2014;20(2): O83-9. doi:10.1111/1469-0691.12337

40. Das K, Bhattacharyya A, Chandy M, et al. Infection control challenges of infrequent and rare fungal pathogens: lessons from disseminated Fusarium and Kodamaea ohmeri infections. Infect Control Hosp Epidemiol. 2015;36(7):866-868. doi:10.1017/ice.2015.103

41. Paul S, Kannan I. Molecular identification and antifungal susceptibility pattern of Candida species isolated from HIV infected patients with candisiasis. Curr Med Mycol. 2019;5(1):21-26. doi:10.18502/ cmm.5.1.533

42. Ioannou P, Papakitsou I. Kodamaea ohmeri infections in humans: a systematic review. Mycoses. 2020;63(7):636-643. doi:10.1111/ myc. 13094

43. Paramythiotou E, Frantzeskaki F, Flevari A, Armaganidis A, Dimopoulos G. Invasive fungal infections in the ICU: how to approach, how to treat. Molecules. 2014;19(1):1085-1119. doi:10.3390/molecules 19011085

44. Harrington R, Kindermann SL, Hou Q, Taylor RJ, Azie N, Horn DL. Candidemia and invasive candidiasis among hospitalized neonates and pediatric patients. Curr Med Res Opin. 2017;33(10):1803-1812. doi:10.1080/03007995.2017.1354824

45. Tóth R, Nosek J, Mora-Montes HM, et al. Candida parapsilosis: from genes to the bedside. Clin Microbiol Rev. 2019;32(2):e0011118. doi:10.1128/CMR.00111-18

46. Kurtzman CP, Smiley MJ. Heterothallism in Pichia kudriavzevii and Pichia terricola. Antonie Van Leeuwenhoek. 1976;42(3):355-363. doi:10.1007/BF00394135

47. Padovan ACB, Rocha WPDS, Toti ACM, Freitas de Jesus DF, Chaves GM, Colombo AL. Exploring the resistance mechanisms in Trichosporon asahii: triazoles as the last defense for invasive trichosporonosis. Fungal Genet Biol. 2019;133:103267. doi:10.1016/j.fgb.2019.103267

48. Pérez-Hansen A, Lass-Flörl C, Lackner M; Rare Yeast Study Group. Antifungal susceptibility profiles of rare ascomycetous yeasts. J Antimicrob Chemother. 2019;74(9):2649-2656. doi:10.1093/jac/ $\mathrm{dkz} 231$ 
49. Jahanshiri Z, Manifar S, Moosa H, et al. Oropharyngeal candidiasis in head and neck cancer patients in Iran: species identification, antifungal susceptibility and pathogenic characterization. J Mycol Med. 2018;28(2):361-366. doi:10.1016/j.mycmed.2018.01.001

50. Wu CJ, Lee HC, Yang YL, et al. Oropharyngeal yeast colonization in HIV-infected outpatients in southern Taiwan: CD4 count, efavirenz therapy and intravenous drug use matter. Clin Microbiol Infect. 2012;18(5):485-590. doi:10.1111/j.1469$0691.2011 .03655 \mathrm{x}$
51. Bensadoun RJ, Patton LL, Lalla RV, Epstein JB. Oropharyngeal candidiasis in head and neck cancer patients treated with radiation: update 2011. Support Care Cancer. 2011;19(6):737-744. doi:10.1007/s00520-011-1154-4

52. Suryawanshi H, Ganvir SM, Hazarey VK, Wanjare VS. Oropharyngeal candidosis relative frequency in radiotherapy patient for head and neck cancer. J Oral Maxillofac Pathol. 2012;16 (1):31-37. doi:10.4103/0973-029X.92970

\section{Publish your work in this journal}

Infection and Drug Resistance is an international, peer-reviewed openaccess journal that focuses on the optimal treatment of infection (bacterial, fungal and viral) and the development and institution of preventive strategies to minimize the development and spread of resistance. The journal is specifically concerned with the epidemiology of antibiotic resistance and the mechanisms of resistance development and diffusion in both hospitals and the community. The manuscript management system is completely online and includes a very quick and fair peerreview system, which is all easy to use. Visit http://www.dovepress.com/ testimonials.php to read real quotes from published authors. 